\title{
Increase of the photosensitivity of undoped poly(methylmethacrylate) under UV radiation at $325 \mathrm{~nm}$
}

\author{
D. Sáez-Rodríguez, ${ }^{1}$ K.Nielsen, ${ }^{2}$ O.Bang, ${ }^{2}$ D.J.Webb ${ }^{1}$ \\ ${ }^{1}$ Aston Institute of Photonic Technologies, Aston University, Birmingham, UK \\ ${ }^{2}$ DTU Fotonik, Dept. of Photonics Engineering, Technical University of Denmark, DK- \\ 2800 Kgs. Lyngby, Denmark \\ *Corresponding author:d.saez-rodriguez@aston.ac.uk
}

\begin{abstract}
In this paper we report, for the first time to our knowledge, an increase of the photosensitivity of a microstructured polymer optical fibre (mPOF) made of undoped PMMA due to applied strain during the fabrication of the gratings. In the work, fibre Bragg gratings (FBGs) have been fabricated in undoped PMMA mPOFs with a hexagonal structure of three rings in the inner cladding. Two sets of FBGs were inscribed at two different resonant wavelengths $(827 \mathrm{~nm}$ and $1562 \mathrm{~nm})$ at different strains using an UV He-Cd laser at 325 $\mathrm{nm}$ focused by a lens and scanned over the fibre. We observed an increase of the reflection of the fibre Bragg gratings when the fabrication strain is higher. The photosensitivity mechanism is discussed in the paper along with the chemical reactions that could underlie the mechanism. Furthermore, the resolution limit of the material was investigated.
\end{abstract}

Keywords:Photosensitivity, FBG, Fibre Bragg grating, polymer optical fibre, PMMA

\section{INTRODUCTION}

The photosensitivity of polymers to UV radiation is a key factor in the fabrication of optical devices, such as gratings and waveguides. For this reason it is important to understand the different mechanisms involved in such index changes. One of the most common polymers in the fabrication of such optical devices is poly(methyl methacrylate) (PMMA) because of its good optical properties. It has been used to fabricate both waveguides in films and fibre Bragg gratings in polymer optical fibre (POF) using UV radiation.

The first study of the photosensitivity of undoped PMMA prepared under special oxidative conditions was done by Tomlinson [1] et al. in 1970 and later by Bowden [2] et al. in 1974. Both authors achieved similar results, getting an increase of the density in the irradiated areas under CW UV radiation at $325 \mathrm{~nm}$. However, each proposed a different mechanism responsible for this density increase. Tomlinson proposed an increase of the density because of the photo-crosslinking effect and Bowden explained it as a consequence of the polymerization of the residual monomers, using as initiator peroxides produced during the fabrication process of the PMMA films studied.

The photosensitivity of these PMMA films was low and the inscription time long, which is why the PMMA was later doped to increase photosensitivity and reduce fabrication time with a different range of dopants such as photoinitiators or photoisomers [3,4]. However, doping PMMA can lead to the loss of some of the good optical properties, such as the transparency, besides complicating the fabrication process of the optical devices due to the doping process.

The first study of PMMA photo-degradation was done by Torikai [5,6] et al. in 1990 and 1993 in air and vacuum, respectively. They demonstrated that photo-degradation of PMMA occured at a wavelength of $320 \mathrm{~nm}$, close to that used in our study. Furthermore, they showed that for longer wavelengths the presence of oxygen during UV inscription did not contribute to the photo-degradation effect. No evidence of photo-degradation has been reported before in PMMA under UV radiation at the 325 nm wavelength frequently used for grating fabrication in PMMA based fibre. However, in the scope of polymer science it is well-known that stress in the sample produces an increase of the photo-degradation effect which could be used to reduce the wavelength threshold for photodegradation under UV radiation [7].

In this paper we report, for the first time to our knowledge, an increase of the photosensitivity of a polymer optical fibre made of undoped PMMA due to applied strain during the fabrication of the gratings. In the work fibre Bragg gratings (FBGs) have been fabricated in undoped PMMA microstructured polymer optical fibres (mPOFs) with a hexagonal structure of three rings in the inner cladding. Two sets of FBGs were inscribed at two different resonant wavelength ( $827 \mathrm{~nm}$ and $1562 \mathrm{~nm}$ ) at different strains using an UV He-Cd laser at $325 \mathrm{~nm}$ focused by a lens and scanned over the fibre. We observed an increase of the reflection of the fibre Bragg gratings when the fabrication strain is higher. The photosensitivity mechanism is discussed in the paper along with the chemical reactions that could underlie the mechanism. Furthermore, the resolution limit of the material was investigated. 


\section{FABRICATION AND RESULTS}

\subsection{Microstructured polymer optical fibre fabrication}

The fabrication of the mPOF was made in two steps. First a commercial PMMA rod of $60 \mathrm{~mm}$ diameter was drilled with a three ring hexagonal cladding structure with a pitch of $\Lambda=6.2 \mathrm{~mm}$ and a hole diameter of $\mathrm{d}=2.5 \mathrm{~mm}$. Then the rod was pulled on the drawing tower to produce a cane with $5.4 \mathrm{~mm}$ diameter.. The $\mathrm{mPOF}$ cane was sleeved with three PMMA tubes with outer/inner diameters of $10 / 7,15 / 11$, and 20/16 mm, forming a new preform, which was then drawn to fibre. Figure 1 shows an optical microscope images of the transversal section of the fibre. The average pitch and hole diameter in the fibre are 4.26 and 1.87 microns, respectively, and the external diameter is 135 micron. This results in a relative hole diameter of $\mathrm{d} / \Lambda=0.44$.

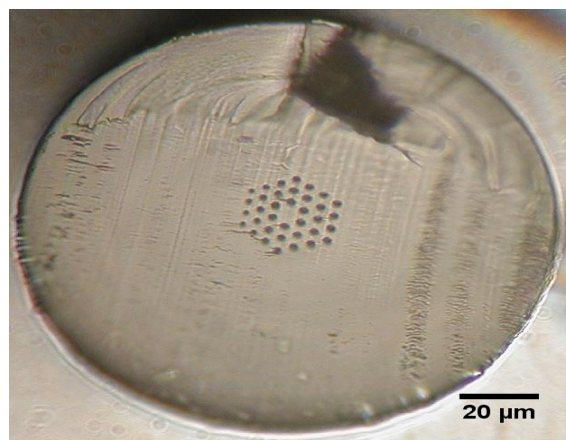

Figure1 Transversal section of the microstructured polymer optical fibre.

\subsection{Fibre Bragg grating inscription.}

A CW He-Cd laser with an output power of $20 \mathrm{~mW}$ at $325 \mathrm{~nm}$ was used to inscribe the FBGs in the mPOF. The inscription was carried out by using a mirror mounted on a motorized translation stage to scan a beam of $1.2 \mathrm{~mm}$ diameter focused with a cylindrical lens (focal length approximately $6 \mathrm{~cm}$ ) along the fibre through the phase mask. The final grating lengths were $1 \mathrm{~cm}$. In order to study the spatial recording resolution of the material two sets of FBGs were inscribed using two different grating pitches (530.59 and 278.75 $\mathrm{nm})$. Each set was fabricated while the fibre was held under a range of different strains during the inscription.

Depending on the orientation of the fibre with respect to the UV beam, the optical power reaching the core can vary significantly due to the hexagonal structure in the cladding of the fibre [8]. In order to evaluate and partially compensate for that, three gratings were inscribed for every inscription strain. In table 1 are shown the gratings' reflectivity (as measured in transmission) for the different inscription strains and for both grating pitches.

\begin{tabular}{|c|c|c|c|c|c|c|}
\hline \multirow{3}{*}{ Strain(\%) } & \multicolumn{6}{|c|}{ Reflectivity (\%) } \\
\hline & \multicolumn{6}{|c|}{ grating pitch $(\mathrm{nm})$} \\
\hline & \multicolumn{3}{|c|}{278.75} & \multicolumn{3}{|c|}{530.59} \\
\hline 0.1 & 4.5 & 0.0 & 1.8 & 0.0 & 0.0 & 0.0 \\
\hline 0.2 & 0.0 & 3.2 & 0.5 & 25.0 & 20.6 & 19.3 \\
\hline 0.3 & 22.0 & 34.2 & 10.1 & 23.4 & 13.3 & 27.6 \\
\hline 0.4 & 28.0 & 2.0 & 3.0 & 39.0 & 35.1 & 21.0 \\
\hline 0.5 & 34.1 & 58.0 & 58.3 & 25.2 & 41.8 & 63.3 \\
\hline 0.6 & 66.0 & 29.0 & 62.2 & 70.0 & 79.0 & 62.0 \\
\hline 0.8 & 48.7 & 25.7 & 29.2 & 80.3 & 56.0 & 99.0 \\
\hline 0.9 & 49.9 & 69.6 & 61.5 & 66.5 & 88.3 & 96.9 \\
\hline 1 & 88.6 & 68.7 & 88.3 & 86.3 & 90.0 & 85.1 \\
\hline
\end{tabular}


Table 1. Reflectivity for the different inscription strains for both grating pitches.

In figure 2 is represented the measurements from table 1 for both phase mask pitches. It can be observed that the reflectivity increases as the inscription strain becomes higher. Also seen is the effect of the microstructure in the fibre on the UV radiation, since there are different values of the reflectivity for each strain inscription.
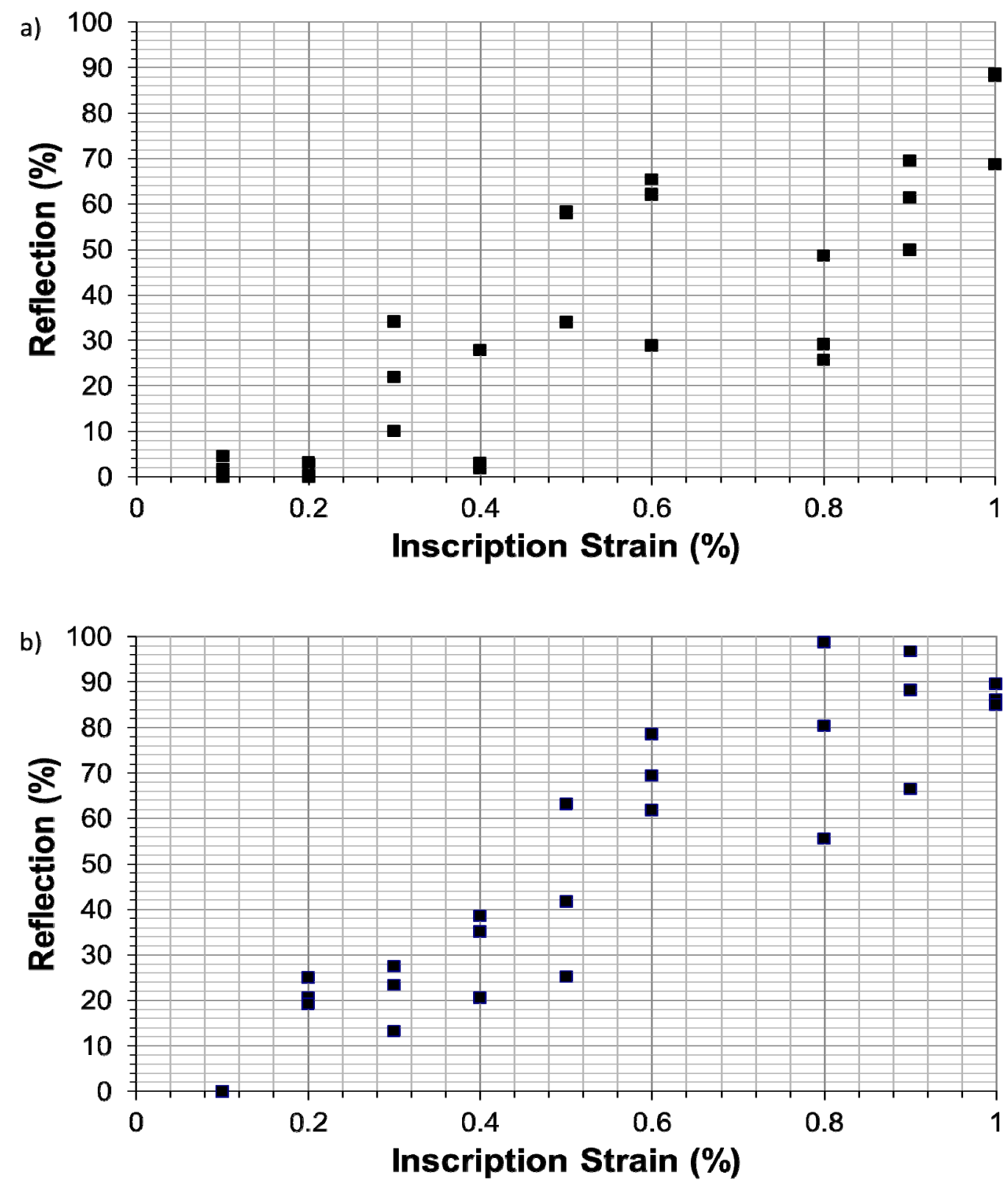

Figure 2 Bragg reflection against inscription strain (a) grating pitch of $278.75 \mathrm{~nm}$ (b) grating pitch $530.59 \mathrm{~nm}$

In figure 3 have been extracted the measurements with highest reflectivity for each strain, which should correspond to the minimum diffraction of UV light from the microstructure in the fibre. 

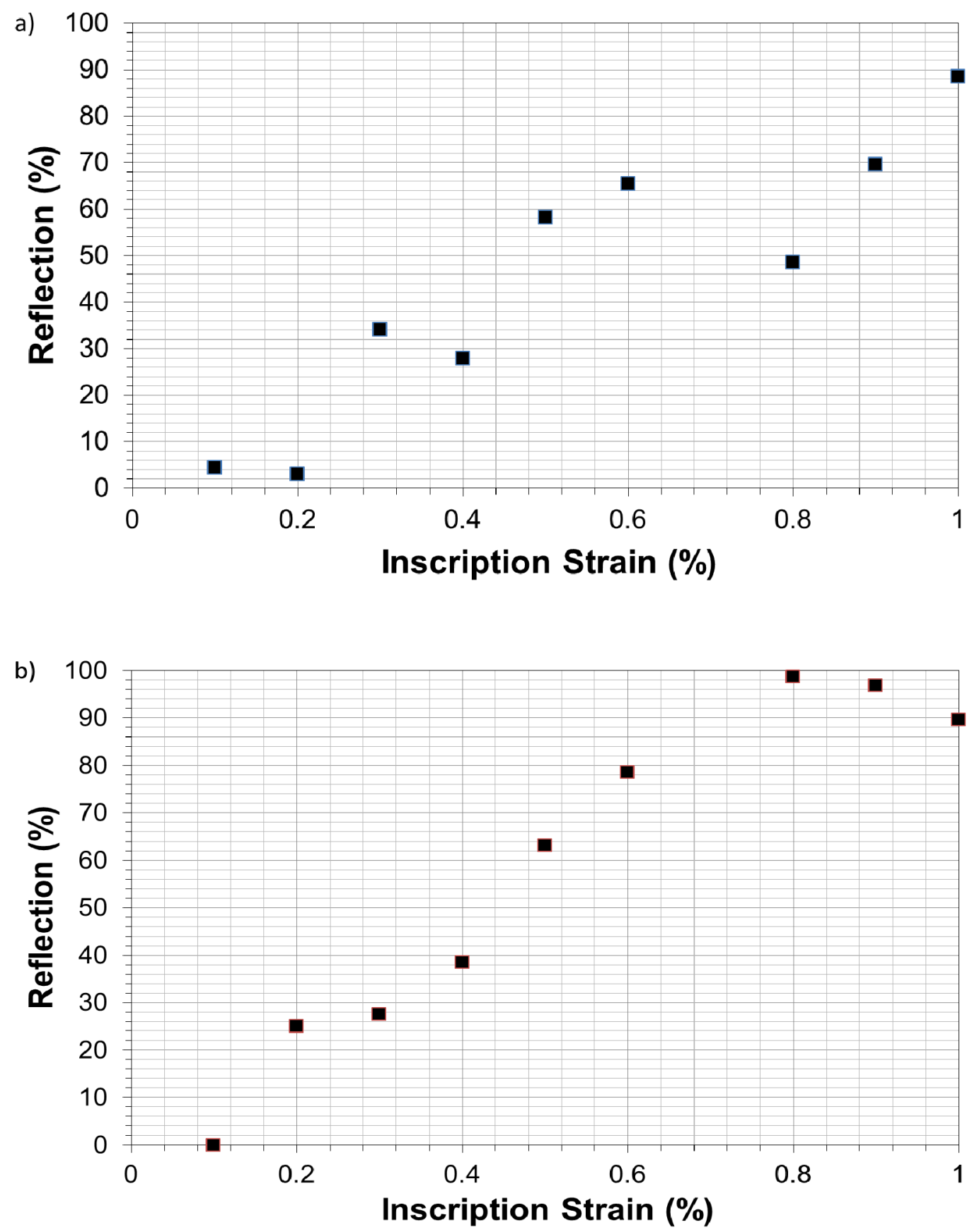

Figure 3 Maximum values of Bragg reflection against inscription strain (a) grating pitch of $278.75 \mathrm{~nm}$ (b) grating pitch $530.59 \mathrm{~nm}$

The refractive index modulation (RIM) of a FBG can be calculated from the experimental parameters (reflectivity, grating length, etc...) of the FBGs inscribed using the equation [9]:

$$
\begin{aligned}
& R=\tanh ^{2}(K \cdot L) \\
& K=\frac{\pi \cdot \Delta n \cdot \eta}{\lambda}
\end{aligned}
$$


where $\mathrm{R}$ is the reflectivity of the grating, $\mathrm{L}$ is the length of the grating, $\mathrm{K}$ is the coupling coefficient, $\Delta \mathrm{n}$ is the index modulation, and $\eta$ represents the fraction of the integrated fundamental mode intensity contained in the core. In the calculation it has been assumed that $\eta=1$ and the effective length of the grating is determined by the $1.2 \mathrm{~mm}$ beam diameter plus the length scanned by the beam $(10 \mathrm{~mm})$, giving a total of $11.2 \mathrm{~mm}$. The RIM as a function of inscription strain for both grating pitches, calculated using the data in figure 3 , is shown in figure 4 .
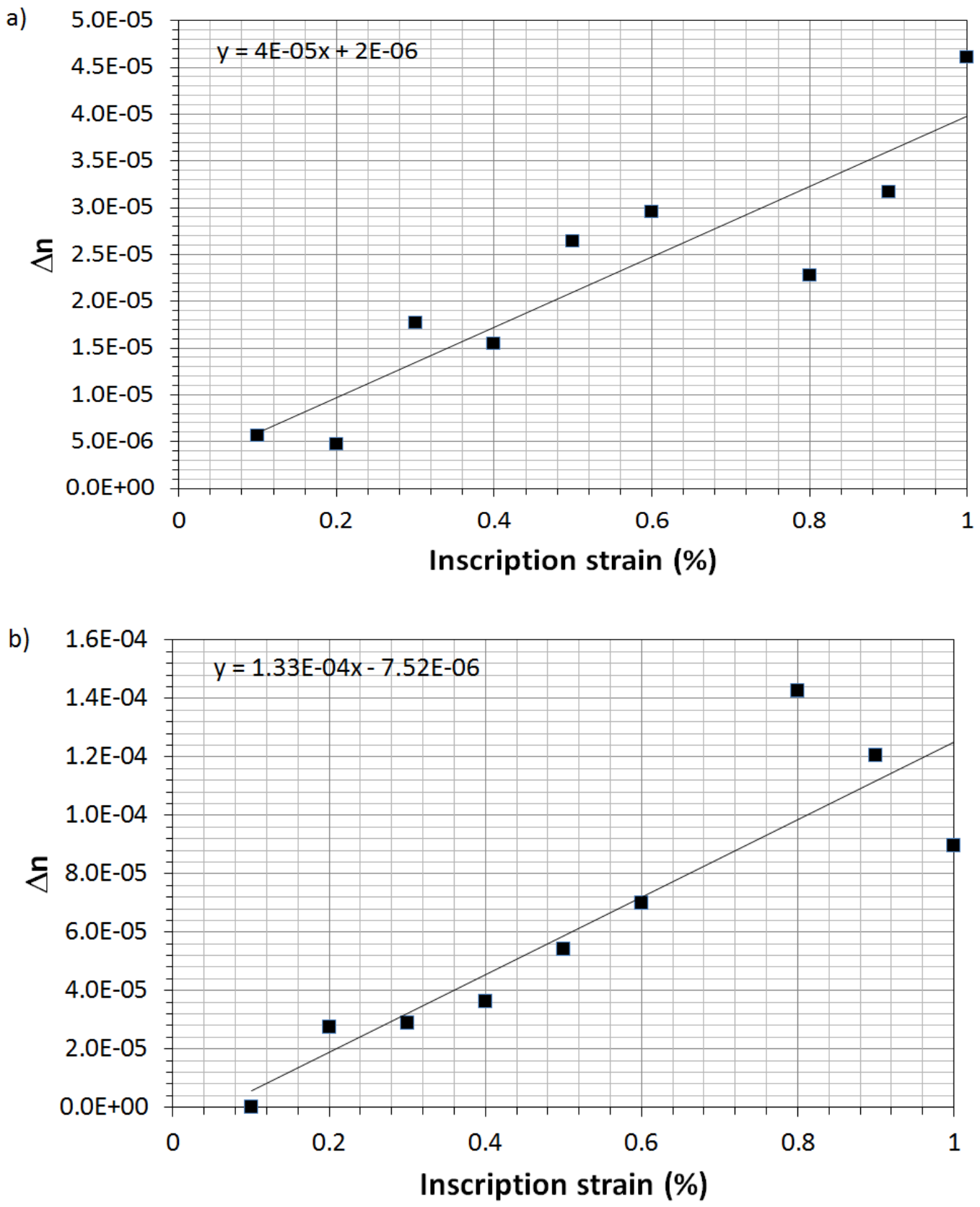

Figure 4 Refractive index Modulation against inscription strain. (a) grating pitch of $278.75 \mathrm{~nm}$, (b) grating pitch of 530.59nm. 


\section{DISCUSSION}

\subsection{Photosensitivity.}

From these results the following was inferred. First, if the grating reflectivity increases with inscription strain and therefore by stress, then there is a concomitant rise in photosensitivity as well. According to [7] the susceptibility to photodegradation in polymers increases with stress, therefore suggesting that the photosensitivity is related to photodegradation.

Secondly, studies of the photodegradation of PMMA irradiated at 260, 280, 300,320, 400 and $500 \mathrm{~nm}$ found that the longest of these wavelengths causing photodegradation was $320 \mathrm{~nm}[5,6]$. The photoreaction responsible for the photodegradation at $320 \mathrm{~nm}$ corresponds to scission of the main chain and the subsequent creation of monomers. Although, those authors say that the photodegradation effect was not observed above $320 \mathrm{~nm}$, the samples were not studied for wavelengths between 320 and $400 \mathrm{~nm}$. In the present work, we used a laser at $325 \mathrm{~nm}$, which we believe is close enough to $320 \mathrm{~nm}$ to enable photodegradation to take place.

\subsection{PMMA resolution.}

Tomlinson [1] et. al. found that their grating period at 0.5 micron was close to the spatial resolution of the PMMA since they measured a refractive index change (RIC) higher than the RIM in the fabricated gratings. In our experiment, we have observed a decrease of the reflectivity of the gratings fabricated with the same inscription strain but with different grating pitch. In figure 3 it could be observed that the reflectivity-strain rate is higher for the grating with large pitch. Moreover, figure 4 shows that the photosensitivity is higher for the larger pitch gratings since the fit has a higher slope in that case. Both results suggest that we are near the spatial resolution limit of the material.

\section{CONCLUSIONS}

We have researched the photosensitivity of undoped PMMA under UV radiation at $325 \mathrm{~nm}$ for different inscription strains; the research provides clues about the photosensitive mechanism of PMMA; according to the results it should be associated with the photo-degradation of the material and in particular with the scission of the main chain and the subsequent creation of monomers. In addition, we noted a decrease of the grating strength, and therefore of the photosensitivity, as the grating pitch become smaller, indicating we are close to the spatial resolution of the material obtaining a similar result to Tomlinson et. al..

\section{ACKNOWLEDGMENTS}

This work was supported by a Marie Curie Intra European Fellowship included in the 7th Framework Program of the European Union (project PIEF-GA-2011-302919).

\section{REFERENCES}

[1] W. J. Tomlinson, I. P. Kaminow, E. A. Chandross, R. L. Fork, W. T. Silfvast, "Photoinduced refractive index increase in poly(methylmethacrylate) and its applications" Appl. Phys. Lett. 16 (12), 486-488 (1970).

[2] M. J. Bowden, E. A. Chandross, I. P. Kaminow, "Mechanism of the photoinduced refractive index increase in Polymethyl Methacrylate" Appl. Opt. Technol. 13 (1), 112-117 (1974).

[3] H. Franke, "Optical recording of refractive-index patterns in doped poly-(methyl-methacrylate) films" Appl. Opt. 23 (16), 2729-2733 (1984).

[4] J-M. Yu, X-M. Tao, H-Y. Tam, D-X. Yang, M.S. Demokan, "Photosensitivity and grating development in trans-4-stilbenemethanol-doped poly(methyl methacrylate) materials" Opt. Com. 265 (1), 132-139 (2006).

[5] A. Torikai, M. Ohno, K. Fueki,"Photodegradation of Poly(methyl Methacrylate) by monochromatic light: quantum yield, effect of wavelengths and light intesity" J. of Applied Polymer Science, 41, 1023-1032 (1990).

[6] T. Mitsuoka, A. Torikai, K. Fueki, "Wavelength sensitivity of the photodegradation of poly(methyl methacrylate" J. of Applied Polymer Science, 47, 1027-1032 (1993).

[7] D. R. Tyler, "Mechanistic aspects of the effects of stress on the rates of photochemical degradation reactions in polymers”J. of Macromolecular Science Part C: Polymer review, 44 (4), 351-388, (2004).

[8] H. Dobb, D. J. Webb, K. Kalli, A. Argyros, M. C. J. Large, M. A. V. Eijkelenborg, "Continuous wave ultraviolet lightinduced fiber bragg gratings in few- and single-mode microstructured polymer optical fibers" Opt. Lett. 30 (24), 32963298 (2005).

[9] A. Othonos, K. Kalli, Fiber Bragg Grating: Fudamentals and Applications in telecommunications and sensing. (1999 Artech House, INC.). 